\title{
Alois Riklin
}

\section{Die Ringparabel und das Projekt Weltethos ${ }^{1}$}

Wer kennt nicht die Ringparabel im Drama Nathan der Weise von Gotthold Ephraim Lessing aus dem Jahr 1779? Doch Lessing ist nicht ihr Erfinder. Er selbst nennt als Quelle eine Novelle im Decamerone von Giovanni Boccaccio (um 1350). Dieser wiederum hatte sie - direkt oder indirekt - dem Novellino entnommen, einer Florentiner Novellensammlung aus dem 13.Jahrhundert. Der Ursprung der Parabel ist jedoch älter. Ihr frühester Beleg, dort freilich als Perlengeschichte, findet sich in einem Dialog zwischen einem Moslem und einem Christen, dem Kalifen Al-Mahdi von Bagdad und dem syrischen Patriarchen Timotheus I. (um 781).

\section{Boccaccio: Decamerone}

Verkürzt lautet die Parabel bei Boccaccio so: Sultan Saladin, der muslimische Herrscher über Jerusalem, versucht, den Juden Melchisedek aufs Glatteis zu führen, indem er von ihm wissen will, welche Religion die wahre sei: die jüdische, die christliche oder die islamische. Der schlaue Jude erkennt die Falle. Um ihr zu entgehen, erzählt er die Geschichte von den drei Ringen. Darin geht es um einen Brauch, wonach seit Generationen der Vater jeweils seinem liebsten Sohn einen mit Wunderkraft versehenen Ring vermacht und ihn damit zugleich zu seinem Erben und zum Oberhaupt der Familie bestimmt. Diese Kette bleibt ununterbrochen, bis ein Vater drei Söhne hat, die er alle gleich liebt. Um keinen bevorzugen zu müssen, lässt er heimlich zwei Kopien anfertigen, die dem echten Ring so sehr gleichen, dass selbst der Vater sie kaum voneinander unterscheiden kann. Bevor er stirbt, schenkt er jedem der drei Söhne einen Ring und lässt jeden im Glauben, den echten geerbt zu haben; so wie Juden, Christen und Muslime je glauben, die wahre Religion zu besitzen. Saladin ist von der Geschichte und von der Klugheit Melchisedeks so beeindruckt, dass er ihm eine hohe und ehrenvolle Stellung an seinem Hof verleiht.

Ort der Handlung, sowohl bei Boccaccio als auch bei Lessing, ist Jerusalem zur Zeit der Kreuzzüge um 1200. Die einzige historische Gestalt in beiden Texten ist Saladin, der Sultan von Aegypten und Syrien, der nunmehr auch über Jerusalem herrschte, nachdem er es von den Christen zurückerobert hatte. Selbst im christlichen Mittelalter galt der siegreiche Feldherr als gerechter Fürst. Für seine religiöse Toleranz spricht die Tatsache, dass er dem bedeutendsten jüdischen Gelehrten des Mittelalters, Moses Maimo-

1 Der Text ist die gekürzte Fassung eines Vortrags im Rahmen der Weltethos-Wochen an der Universität Freiburg i.Ue. (Mai 2008). 
nides, nach dessen Vertreibung aus Cordoba an seinem Hof Asyl gewährte. Dante platzierte Saladin in der Divina Commedia immerhin in die »Vorhölle«, zusammen mit den guten »Heiden«. Und im Convivio wählte er ihn als Vorbild für die Tugend der Freigebigkeit.

\section{Lessings Doppeldrama}

Lessing hat aus der Novelle von Boccaccio ein Doppeldrama gestaltet: ein Familiendrama und ein Religionsdrama. Die fünf Hauptpersonen des Familiendramas sind: die Moslems Saladin und seine Schwester Sittah, der Jude Nathan, das Mädchen Recha und ein christlicher Tempelherr. Sie sind durch eine tragische Vorgeschichte miteinander verbunden. Saladin und Sittah trauern um ihren in den kriegerischen Auseinandersetzungen verschollenen Bruder. Weil ihm der christliche Tempelherr ähnlich sieht, hat ihn Saladin begnadigt. Nathans Frau und seine sieben Söhne sind von Christen ermordet worden. Er selbst wurde von einem christlichen Kreuzfahrer gerettet. Dieser hat ihm später die kleine Recha als Pflegekind anvertraut, bevor er in den Kampf zog und darin umkam. Nathan liebt das Mädchen wie sein eigenes Kind. Der christliche Tempelherr hat Recha aus dem brennenden Haus des abwesenden Nathan in Sicherheit gebracht, worauf sich die beiden ineinander verlieben.

Die fünf Hauptpersonen sind, ohne es zu wissen, miteinander verwandt. Der verschollene Bruder von Saladin und Sittah hatte nämlich eine Christin aus dem Haus der Staufer geheiratet. Dieser muslimisch-christlichen Mischehe entsprossen Recha und der christliche Tempelherr. Sie sind also Geschwister, und sie sind Nichte und Neffe Saladins und Sittahs. Das Geheimnis der unwahrscheinlichen, aber in den Wirren der Kreuzzüge nicht unmöglichen Verwandtschaft wird erst in der Schlussszene des Dramas enthüllt. Unmittelbar bevor der Vorhang fällt, schliessen sich Jude, Christen und Moslems friedlich vereint in die Arme. Die Botschaft ist klar: Alle drei Religionen bilden als Abkömmlinge des Stammvaters Abraham eine Familie, verbunden durch den Glauben an den einen und gleichen Gott.

Das Religionsdrama muss vor dem Hintergrund der religionspolitischen Positionen Lessings gedeutet werden. Der theologisch gebildete Philosoph und Literat lehnte den im Christentum seit dem Mittelalter vorherrschenden Anti-Judaismus und Anti-Islamismus ab. Die Juden galten vielen „Rechtgläubigen“ als Christusmörder, den Koran hielten sie für ein Lügenbuch und Mohammed für einen Diener des Teufels. Man kann sich das Entsetzen Lessings vorstellen, als er auf seiner Italienreise das päpstliche Edikt über die Juden (1775) las. Darin verbot Pius VI. unter Strafandrohung, dass Christen und Juden miteinander spielen, essen, trinken oder reden. Er untersagte den Juden, Christen anzustellen, und er gebot den Rabbinern, ihre Gläubigen der christlichen Predigt zuzuführen. Der Lutheraner Lessing wusste freilich, dass Martin Luther in Sachen Antisemitismus um keinen Deut milder war. Dennoch lehnte er den Religionsverzicht zu Gunsten eines säkularen Humanismus ab, wie das Voltaire kurz zuvor gefordert hatte. Der französische Aufklärer verurteilte das Christentum als Unheil stiftende Religi- 
on, die Juden als das »abscheulichste Volk « und Mohammed als Fanatiker. Sein »Ecrasez l'infâme« richtete sich im Grunde gegen jede Religion. Auch eine Vermischung der Religionen, eine Art Universalreligion, kam für Lessing nicht in Frage. Vor allem verurteilte er die wechselseitige Rechthaberei, so als ob die je eigene Religion im Alleinbesitz der Wahrheit wäre.

Aus der Sicht Lessings gibt es auf Erden keine religiöse Wahrheitsgewissheit. Die Wahrheit bleibt ein ungelöstes »Rätsel«. Dennoch empfahl er den Juden, Christen und Muslimen, an der angestammten Religion festzuhalten. Dieses unbeirrte Festhalten ist für Nathan eine Frage von »Treu und Glauben« gegenüber den eigenen Vorfahren und der eigenen Geschichte. Er sieht keinen vernünftigen Grund, warum ein Jude seinen Vätern weniger glauben sollte als ein Christ oder ein Moslem den seinen. Als Vision schwebte Lessing ein friedliches Neben- und Miteinander der drei abrahamischen Religionen vor. Sein Drama Nathan der Weise lässt das interreligiöse Konfliktpotential hinter sich und weist den Weg zur Versöhnung in gegenseitigem Respekt.

Im ersten Teil seiner Ringparabel blieb Lessing noch nahe an der Novelle von Boccaccio, mit zwei Ausnahmen: Im Gegensatz zum Decamerone sind die drei Ringe selbst für den Vater nicht unterscheidbar, und in Nathans Erzählung wird dem echten Ring die geheime Kraft zugeschrieben, »vor Gott und Menschen angenehm zu machen«.

\section{Lessings Lösung}

Der zweite Teil der Parabel hat mit Boccaccios Vorlage nichts mehr zu tun. Vielmehr setzt Lessing die Geschichte fort: Kaum ist der Vater tot, geraten die Brüder in Streit. Dieser Streit steht für die Jahrtausendkonflikte zwischen den drei Religionen und ihren konfessionellen Untergliederungen, mit Kreuzzügen, kriegerisch-aggressiver Interpretation des Jihad-Begriffs, Religionskriegen, Konfessionskriegen, Massenmorden, Zwangsmissionierungen, Umsiedlungen, Massenvertreibungen, Enteignungen, Inquisition, Folter, Scheiterhaufen, Ketzer- und Hexenprozessen. Dabei diente die Religion oft nur als Vorwand für politischen Machtgewinn, wirtschaftliche Ausbeutung und persönlichen Vorteil.

Der religiös verbrämte Machtmissbrauch von weltlichen und geistlichen Potentaten, die Leichtverführbarkeit der Menschen durch Personenkult und Karrierechancen sowie ihre Anfälligkeit für konstruierte oder aufgebauschte Feindbilder sind vielfach bestätigte Tatsachen, und dies bis zum heutigen Tag. Den Fundamentalismus, auch den gewalttätigen, gab und gibt es in der Praxis von Anhängern aller abrahamischen Religionen. Der religiös verwirrte, ohnmächtige Terrorismus von unten ist das Spiegelbild des mächtigen, mitunter auch religiös verschleierten inner- und zwischenstaatlichen Staatsterrorismus von oben. Die Opfer sind allemal vor allem Unschuldige, die sich zufällig zur falschen Zeit am falschen Ort befinden.

Immerhin, es gab auch Zeiten und Orte friedlichen Zusammenlebens der abrahamischen Religionen, vor allem im südlichen Europa und im Vorderen Orient, zum Beispiel im maurischen Andalusien, im normannischen Sizilien, in Jerusalem zur Zeit Sala- 
dins und des Stauferkönigs Friedrich II. oder in Livorno zur Zeit von Lessings Italienreise. Daran knüpfte Lessing an.

In der Fortsetzung der Parabel soll ein Richter entscheiden, wer von den drei Brüdern den echten Ring erhalten hat und folglich der »Fürst des Hauses« ist. Diese Einführung eines Richters war nicht neu. Er findet sich sowohl in der Ringparabel des Dominikaners Etienne de Bourgon als auch in der Perlengeschichte des jüdischen Mystikers Abraham Abulfia (beide 13.Jahrhundert). Wahrscheinlich kannte Lessing die eine oder andere Erzählung. Wenn er sie kannte und diese zweite Quelle im Gegensatz zur ersten von Boccaccio dennoch nicht preisgab, liegt die Erklärung nahe: Weil die Richter in beiden Vorläufern exklusiv urteilen, nämlich zu Gunsten des Christentums bzw. des Judentums. Genau dies passte Lessing nicht.

Stellvertretend für die Nachkommen Abrahams erteilt der Richter den drei Brüdern zunächst eine gehörige Standpauke. Dann aber gibt Lessing der Geschichte eine überraschende Wendung: Möglich, dass der Vater »die Tyrannei des Einen Rings“« nicht länger dulden wollte; gewiss, dass er alle drei Erben geliebt und gleich geliebt hat. So glaube denn jeder, im Besitz des echten Rings zu sein und halte der Religion seiner Vorfahren die Treue. »Wohlan! Es eifre jeder seiner unbestochenen, von Vorurteilen freien Liebe nach! Es strebe von euch jeder um die Wette, die Kraft des Steins in seinem Ring an Tag zu legen! komme dieser Kraft mit Sanftmut, mit herzlicher Verträglichkeit, mit Wohltun, mit innigster Ergebenheit in Gott zu Hülf'! Und wenn sich dann der Steine Kräfte bei euren Kindes-Kindeskindern äussern: So lad ich über tausend tausend Jahre sie wiederum vor diesen Stuhl. Da wird ein weisrer Mann auf diesem Stuhle sitzen als ich, und sprechen.«

Als ob ihm ein spontaner Einfall gekommen wäre, spielt Nathan den Ball geschickt ins Feld von Saladin zurück: Seid Ihr vielleicht der weisere Richter? Saladin ist zutiefst betroffen, beschämt, gerührt, erschüttert: »Ich Staub! Ich Nichts! O Gott! (...) Nathan, lieber Nathan! - Die tausend tausend Jahre deines Richters sind noch nicht um. Sein Richterstuhl ist nicht der meine. - Geh! - Geh! - Aber sei mein Freund.«

\section{Lessings Lücke}

Die Lösung Lessings war meinem hochgeschätzten und vergleichsweise liberalen Deutschlehrer im Benediktinerkolleg dann doch zu unorthodox. Das war freilich noch bevor das II.Vatikanische Konzil die Religionsfreiheit anerkannte sowie das Wahrheits- und Heilspotential auch in anderen Religionen konzedierte. Angesichts des Unheils, das der absolute Wahrheits- und Heilsanspruch aller drei Glaubensbekenntnisse im Lauf der Geschichte angerichtet hat, scheint der Vorschlag Lessings auf den ersten Blick vernünftig. Aber auf den zweiten Blick ist er, obwohl vernünftig, nicht hinreichend. Warum? Weil der interreligiöse Wettbewerb ohne ergänzende Vorkehren zum Wettstreit verkommen kann, wenn jeder der Bessere, Frömmere, Gottergebenere, Gottgefälligere, Auserwähltere sein will, auch wenn das nicht der Intention Lessings entsprach. 
Wie kann die Lücke bei Lessing geschlossen werden? Es braucht Rahmenbedingungen, stärkere Rahmenbedingungen als nur den Appell für die Tugenden von Liebe, Sanftmut, Verträglichkeit, Wohltun und Gottergebenheit. Und es braucht heute globalere Rahmenbedingungen an Stelle der Beschränkung auf die drei monotheistischen Religionen. So wie der sportliche Wettkampf auf dem Fussballplatz ohne die Einhaltung von Spielregeln und ohne die Bändigung fanatisierter Fans ausartet, so wie der wissenschaftliche, politische oder wirtschaftliche Wettbewerb ohne mässigende Rahmenbedingungen schädlich ist, so bedarf auch der inter- und innerreligiöse Wettbewerb hinreichender ethischer Rahmenbedingungen. Welche? Hier kann das Projekt Weltethos von Hans Küng weiterhelfen.

\section{Rabmenbedingungen: Projekt Weltethos}

Hans Küng und sein Kollege Karl-Josef Kuschel haben sich intensiv mit der Ringparabel Lessings auseinandergesetzt, ohne meines Wissens das Projekt Weltethos explizit mit ihm in Verbindung zu bringen. In diesem Projekt ist Lessings Ansatz weiterentwickelt, von den drei abrahamischen Religionen auch zu anderen Religionen und darüber hinaus auch zu nicht religiös begründeten Friedenskonzepten. Vor allem aber erfüllen die Forderungen des Projekts nach einem Dialog zwischen den Religionen, nach Betonung der Gemeinsamkeiten statt des Trennenden und nach einem minimalen Konsens globaler ethischer Standards jene Rahmenbedingungen, die geeignet scheinen, die von Lessing hinterlassene Lücke zu schliessen.

Damit soll nicht unterstellt werden, das Projekt Weltethos sei die einzige derartige Initiative. Aber es hat weltweit unbestreitbar die stärkste Ausstrahlung erlangt, von der Lancierung (1990) über die Erklärung des Parlaments der Weltreligionen von Chicago (1993) und die Menschenpflichten-Erklärung des InterAction Council ehemaliger Staats- und Regierungschefs (1997) bis zur Resolution Globale Agenda für den Dialog der Kulturen der UN-Generalversammlung (2001). Keine andere Initiative ist durch eine so grundlegende Religionsforschung unterbaut, die Trilogie von Hans Küng über Judentum (1991), Christentum (1994) und Islam (2004), sein Sachbuch zur siebenteiligen Filmreihe Spurensuche. Die Weltreligionen auf dem Weg (1999), das Werk von Stephan Schlensog über den Hinduismus (2006) und die beiden Bücher von Karl-Josef Kuschel über die abrahamischen Religionen (2001, 2007). Keine andere Initiative hat die Konkretisierung globaler ethischer Standards für Politik und Wirtschaft so weit vorangetrieben, mit Küngs Weltethos für Weltpolitik und Weltwirtschaft (1997), mit Markus Weingardts Beschreibung und Analyse von vierzig Fallbeispielen zum Friedenspotential der Religionen (2007) und mit Hans-Martin Schönherr-Manns Zusammenschau von fünfzehn universalethischen Ansätzen in der Philosophie des 20.Jahrhunderts (2008). Keine andere Initiative hat so umfassende pädagogisch-didaktische Materialien zur Verbreitung der Weltethos-Idee bereitgestellt (Ausstellung, Lehrmittel, interaktives Internet-Lernprogramm usw.). Und schließlich hat keine andere Initiative so viele (um die hundert) weiterführende Stellungnahmen von Repräsentanten verschiedener Religio- 
nen, Wissenschaftlern aller Fakultäten, Kulturträgern, Wirtschaftsführern, Politikern und Friedensnobelpreisträgern eingeholt und pupliziert.

Im Sinne des Projekts Weltethos sollten für den erweiterten Lessing'schen Wettbewerb etwa die folgenden Rahmenbedingungen gelten: Selbstkritik vor Fremdkritik Wahrhaftigkeit - Absage an jeden exklusiven Wahrheits- und Heilsanspruch - gegenseitiger Respekt (also mehr als nur Toleranz) - Dialog der Religionen und Kulturen Religionsfreiheit - Gewaltlosigkeit (mit legitimen Ausnahmen) - Solidarität mit den Benachteiligten - Gleichberechtigung von Mann und Frau. Gegenüber solchen Rahmenbedingungen haben wohl alle Religionen noch Nachholbedarf, bestimmt aber die drei abrahamischen.

\section{Verschwiegene Quelle}

Bleibt eine letzte Frage: Lessing hat seine erste Quelle offengelegt. Die zweite - jene für den Richter - hat er verschwiegen. Gibt es vielleicht noch eine dritte Quelle, die er nicht preisgeben wollte? Gemeint ist eine Vorgabe für die Idee des interreligiösen Wettbewerbs zum Guten. Etwa die Stelle im Neuen Testament: An den Früchten werdet ihr sie erkennen, die falschen und die wahren Propheten? (Mt 7, 15-20)? Noch passender, ja eigentlich deckungsgleich, ist ein anderer verblüffender Text, den Lessing auf Grund der von ihm benutzten englischen Übersetzung zweifellos gekannt hat: „Für jeden von euch (Juden, Christen und Moslems - A. R.) haben Wir eine Richtung und einen Weg festgelegt. Und wenn Gott gewollt hätte, hätte Er euch zu einer einzigen Gemeinschaft gemacht. Doch will Er euch prüfen in dem, was Er euch hat zukommen lassen. So eilt zu den guten Dingen um die Wette. Zu Gott werdet ihr allesamt zurückkehren, dann wird Er euch kundtun, worüber ihr uneins wart.“

Die Quelle ist: Koran, Sure 5, 48.

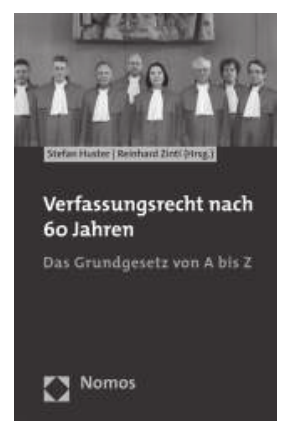

\section{Verfassungsrecht nach 60 Jahren}

Das Grundgesetz von A bis $Z$ Herausgegeben von Stefan Huster und Reinhard Zintl 2009, 222 S., brosch., 19,90€, ISBN 978-3-8329-4391-2

Bitte bestellen Sie im Buchhandel oder versandkostenfrei unter $\longrightarrow$ www.nomos-shop.de

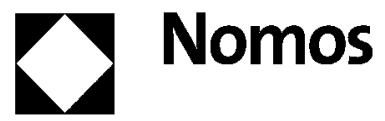

ZfP 56. Jg. 2/2009 